\title{
Evaluation of a novel low-cost disposable endoscope for visual assessment of the esophagus and stomach in an ex-vivo phantom model
}

\section{다(1)우우}

\author{
Authors \\ Institutions \\ 1 Mechanical Engineering Department, Vanderbilt \\ University, Nashville, Tennessee, United States \\ 2 Division of Internal Medicine, Vanderbilt University \\ Medical Center, Nashville, Tennessee, United States \\ 3 School of Electronic and Electrical Engineering, \\ University of Leeds, Leeds, United Kingdom \\ 4 Division of Gastroenterology, Vanderbilt University \\ Medical Center, Nashville, Tennessee, United States
}

Nicolò Garbin ${ }^{1}$, Alexander P. Mamunes², Dennis Sohn ${ }^{1}$, Ryan W. Hawkins ${ }^{1}$, Pietro Valdastri1,3, Keith L. Obstein ${ }^{1,4}$

\section{ABSTRACT} ness of the platform are unknown. submitted 14.12 .2018

accepted after revision 16.3.2019

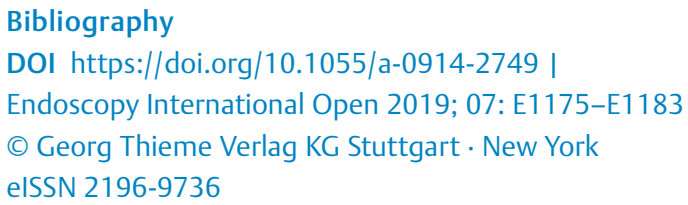

Corresponding author

Keith L. Obstein, MD, MPH, FASGE, FACG, AGAF, 1301 Medical Center Drive, Suite 1660 TVC, Nashville, TN 37232

Fax: $+1-615-343-8174$

keith.obstein@vumc.org

Supplementary Table 1 and 2

Online content viewable at:

https://doi.org/10.1055/a-0914-2749
Background and study aims Our academic lab has developed a novel, low-cost, disposable endoscope for assessment of the esophagus and stomach without need for large equipment or complex electronics. Usability and intuitive-

Methods The novel endoscope (NE) consists of a high-definition camera, LED module, and three bellows. Compressed air actuates the bellows, producing camera/LED articulation. Insufflation and lens cleaning ports are present. Video can be displayed on any monitor. Total material costs less than \$35 US. Five novices, five fellows, and five attendings performed five trials using a conventional endoscope and the NE on an upper tract phantom with six gastric landmarks marked. Outcomes included successful identification and time to landmarks; and intuitiveness (NASA task load index; user comments).

Results All landmarks were successfully identified with both endoscopes for all trials $(n=900)$. Attendings and fellows were quicker with the conventional endoscope when compared to the NE $(24.48 \vee 37.13$ s; $P<0.01)$. There was no significant time difference between platforms for novices $(P=0.16)$. All users found the NE intuitive with low mental and physical demand. Novices reported lower temporal demand and effort when using the NE.

Conclusions The NE was easy to maneuver, intuitive, and successful at visualizing gastric landmarks. All users were pleased with the NE drive mechanism and were successful at visualizing the gastric landmarks in a clinically acceptable time. The novel platform has the potential to facilitate rapid, low-cost, diagnostic assessment of the esophagus and stomach in non-traditional settings - facilitating patient management decisions, minimizing encumbrance, and avoiding cross-contamination.

\section{Introduction}

Upper endoscopy, or esophagogastroduodenoscopy (EGD), is a commonly performed procedure allowing for direct visualization (diagnostic) and interaction (therapeutic) with mucosal surfaces of the esophagus, stomach, and first part of the small intestine. Common indications include evaluation of gastrointestinal bleeding, dysphagia, gastroesophageal esophageal reflux disease (GERD) non-responsive to medical therapy, esophageal varices screening, and anemia [1]. The utility of EGD in the evaluation and management of many upper gastrointestinal (UGI) conditions has made it an integral part of patient care. 
Unfortunately, despite the large number of EGDs performed annually in the world, access to the procedure remains limited as EGD is typically performed in specialized centers (ambulatory surgery centers) and select locations within a hospital (endoscopy lab, intensive care unit [ICU], emergency department $[E D])$. The main reasons for these limitations relate to the need for sedation (which requires monitors, intravenous, rescue equipment, oxygen, staff, recovery time) and large, cumbersome, expensive, bulky equipment (endoscopy light source and processor, endoscope, monitor, cables, cart, computer) with intricate handling, maintenance, and reprocessing facilities as current standard. Sedation-related adverse events (AEs) - including hypoxemia, hypotension, and aspiration pneumonia - are the most common complications associated with endoscopy (making up $60 \%$ of all EGD AE) [2]. In addition, conventional EGD requires reprocessing of equipment with risk of cross-contamination or infection. Each used endoscope undergoes leak testing, manual cleaning, high-level disinfection, and repairs if indicated, with an estimated cost ranging from $\$ 114$ to $\$ 280$ per use [3]. Another limitation to EGD access is the relative shortage of providers able to perform the procedure, especially in rural and remote locales, due to need for specialized training in order to perform the procedure successfully - as a significant amount of time is dedicated to mastering both the technical and cognitive aspects [4].

To overcome these limitations and facilitate a potential paradigm shift in gastrointestinal endoscopy, our team has developed a novel, low-cost, disposable, portable, intuitive platform for diagnostic upper endoscopy that potentially can be used off-the-shelf, at the bedside, in non-traditional settings (i.e. primary care office, hepatology clinic) and in the hospital to facilitate efficient triage and patient care - thereby leaving conventional upper endoscopy, as we currently know it, to tissue sampling and therapy. Our system uses direct mechanical coupling between a mechanical joystick and a pneumatically actuated endoscopic tip, allowing for intuitive handling and manipulation that may permit rapid, low-cost, bedside visualization of the esophagus and stomach by a variety of healthcare providers without a long technical learning curve [5]. System functionality, effectiveness in visualizing key gastric landmarks, intuitiveness of the drive mechanism, and user satisfaction in a human phantom, as a step toward first-in-human use, remains unknown.

The aim of this study was two-fold. First, we aimed to assess if our novel platform provided enough range of motion at the distal tip to allow for successful visualization of key gastric landmarks. Second, we aimed to quantify and compare ease of use and intuitiveness of the novel esophago-gastroscope with a conventional upper endoscope.

\section{Methods}

\section{Platform}

Endoscope

The novel esophago-gastroscope ( $\nabla$ Fig. 1a) is composed of a 13.5-mm-diameter clear tip (Clear V3, FormLabs, Inc., Somerville, Massachusetts, United States) ( $\triangleright$ Fig. 1b) that embeds a $1280 \times 1024$-pixel high-definition camera for imaging and three light-emitting diodes (LEDs) for illumination of the esophagus and stomach. The tip is then connected to three rubber bellows (BC-2305, rubberstore.com, Vandalia, Ohio, United States) arranged in parallel ( $\$$ Fig. 1b) and attached to ports in a 7-mmdiameter highly compliant, flexible, seven-lumen catheter. The catheter allows for wiring, irrigation/lens cleaning, lumen insufflation, and pressurization of the bellows from the operating handle. The operating handle is a multi-backbone continuum joystick ( $\triangleright$ Fig.1c) [6,7] with four embedded Nitinol wires within six plastic disks separated by springs - one centrally located (referred to as the primary backbone) and three equally spaced around it (referred to as the secondary backbones). This structure terminates in a pivoting pad. The novel endoscope weighs a total of $0.56 \mathrm{Kg}$ and when produced en-masse has an inclusive cost of approximately 32 USD per endoscope ( Table 1).

The drive mechanism for the novel endoscope is characterized by direct mechanical coupling between the continuum joystick and the steerable tip. This is achieved by pneumatic actuation via a mechanism known as a Parallel Bellow Actuator (PBA) $[8,9]$. As the joystick is manipulated about the pivoting pad, the free ends of the secondary backbones elongate and retract. By coupling the free ends with three 10 -cc syringe pistons, embedded in the device handle ( $\triangleright$ Fig. 1c), motion is converted to bellow pressurization and depressurization. Variation in pressure directly corresponds to change in bellow length - yielding three degrees of freedom: (1) tilting angle, (2) angle of the tilting plane, and (3) elongation/retraction ( $>$ Fig. 2). This, in turn, directs the tip in a configuration commensurate to the joystick's input configuration.

Similar to a conventional endoscope, the device is operated using one hand to steer the tip and the other for inserting or removing the insertion tube/catheter. The device is designed for ambidextrous use (i.e. it is not hand-specific) as the handle is maneuvered about the pivoting pad secured against the operator's body ( $\triangleright$ Fig. 1a).

Ten AA batteries power the system and video is transmitted to the operator's personal device (i. e. mobile phone, tablet, TV, computer monitory) via a secure local Wi-Fi feed (novel endoscope directly to the personal device). Insufflation, irrigation, and lens cleaning are operated via quick-connect port and 60cc syringe ( $>$ Fig. 1a).

\section{Phantom}

A human stomach and esophagus phantom was created from the computed tomography scan images of a $70-\mathrm{kg}$ adult male patient. The images were extracted and reconstructed to serve as a $3 \mathrm{D}$ printed mold (Ultimaker Inc, Geldermalsen, Nether- 

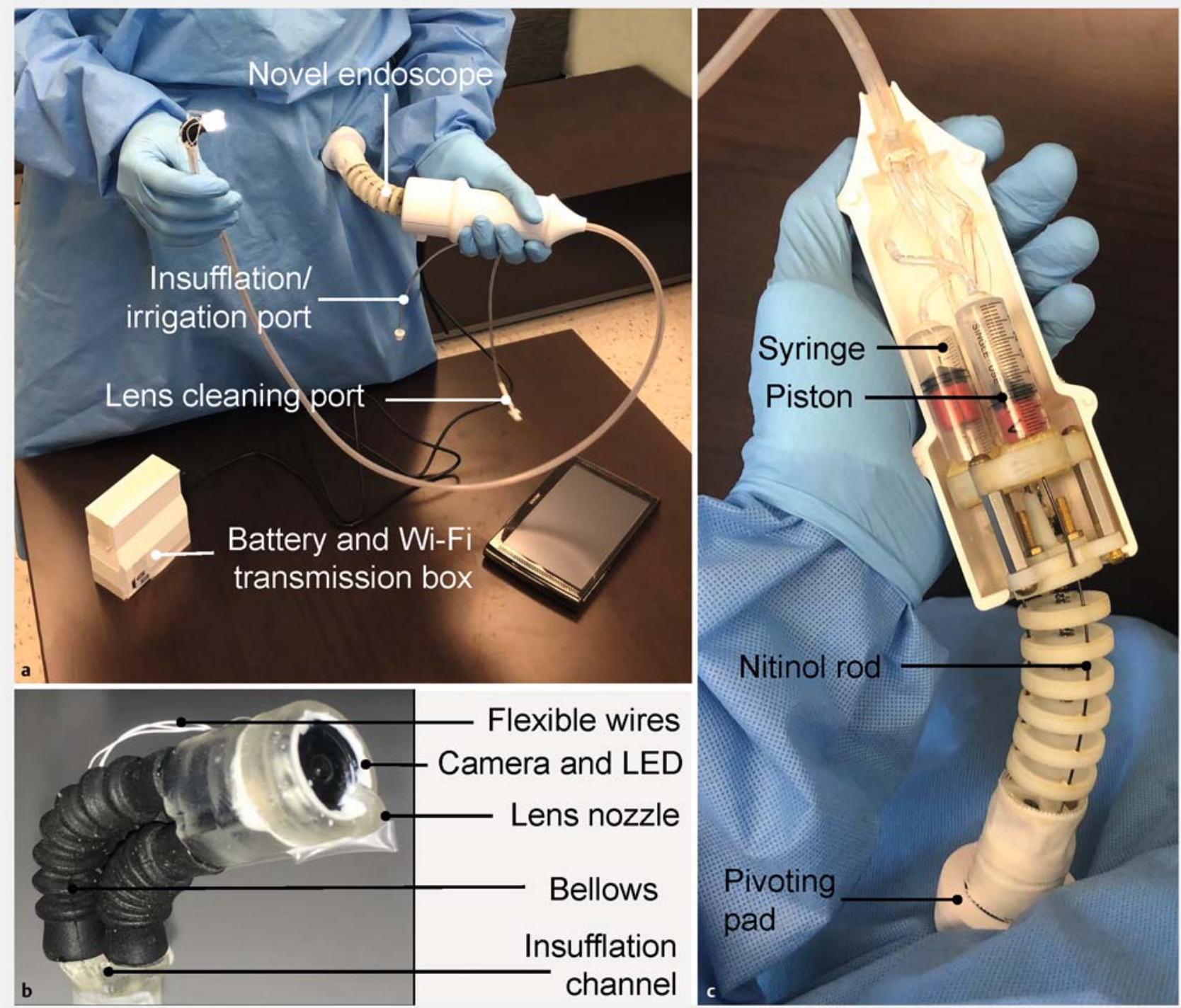

- Fig. 1 The novel disposable flexible endoscope a with close-up view of the tip $\mathbf{b}$ and handle $\mathbf{c}$ (handle cover has been removed to allow visualization of the internal components).

lands). Silicone was then placed in layers to construct the phantom (EcoFlex 00-30, SmoothOn, Macungie, Pennsylvania, United States) ( $\mathbf{F i g . 3}$ ). Ultra-low power LEDs were placed at key anatomic locations within the molded stomach as it was constructed to serve as consistent markers of gastric landmarks. A total of six anatomic landmarks were affixed: gastroesophageal junction (GEJ), fundus, body greater curvature (GC), body lesser curvature (LC), antrum GC, and antrum LC. LED activation was controlled by an embedded micro controller (Arduino Mega 2560, Turin, Italy) connected via USB to a dedicated data-recording computer (OptiPlex 390, Dell, Round Rock, Texas, United States) that allowed the LEDS to be turned on and off during the trial. The phantom was encased in a wood support structure to prevent participant visualization of the landmarks or phantom externally.

\section{Study design}

A total of 15 participants were selected to perform endoscopy in the stomach and esophagus phantom. The participants were selected from our tertiary care medical center by email and face-to-face encounters. Five gastroenterology attendings (> 1000 lifetime conventional upper endoscopies), five gastroenterology fellows (postgraduate year (PGY) 4-6), and five novices (PGY 1 internal medicine residents who had never handled an endoscope) were enrolled. Each user performed endoscopic examination of the GEJ and stomach with the conventional upper endoscope (Karl Storz, Tuttlingen, Germany) and with the novel disposable endoscope. Each participant performed a total of 10 examinations using both devices in random order (conventional $n=5$; novel $n=5 ; 1: 1$ fashion). Examination was performed in a gastroenterology outpatient clinic examination room ( $\triangleright$ Fig. 4 ). Each user was given a 1 -minute practice period 
- Table 1 Novel endoscope component cost based on large-scale production.

\begin{tabular}{|l|c|}
\hline Component & Price (USD) \\
\hline Plastic parts & $\$ 7.00$ \\
\hline Multi-Lumen catheter & $\$ 1.10$ \\
\hline Wiring & $\$ 1.50$ \\
\hline Bellows (3) & $\$ 6.00$ \\
\hline Syringes (3) & $\$ 1.00$ \\
\hline Camera and LED
\end{tabular}

- Fig. 2 The joystick and corresponding tip motion of the disposable flexible endoscope: a elongation, $\mathbf{b}$ retraction, $\mathbf{c}$ tilting angle, $\mathbf{d}$ angle of tilting plane.

with the conventional endoscope and novel endoscope the first time it was selected. The practice period was performed outside of the phantom. After this minute, the user introduced the endoscope into the phantom and positioned the tip in the distal esophagus, $5 \mathrm{~cm}$ from the GEJ. The anatomic landmark LEDs were then illuminated and the user was tasked with identification of all six gastric landmarks ( $\triangleright$ Fig.5). Successful identification of the landmark was denoted by en-face visualization with the endoscope and verbally communicating the color of the LED to the study staff. The time of identification was recorded and the LED was turned off. The exam was considered complete when all six landmarks were successfully identified or if a

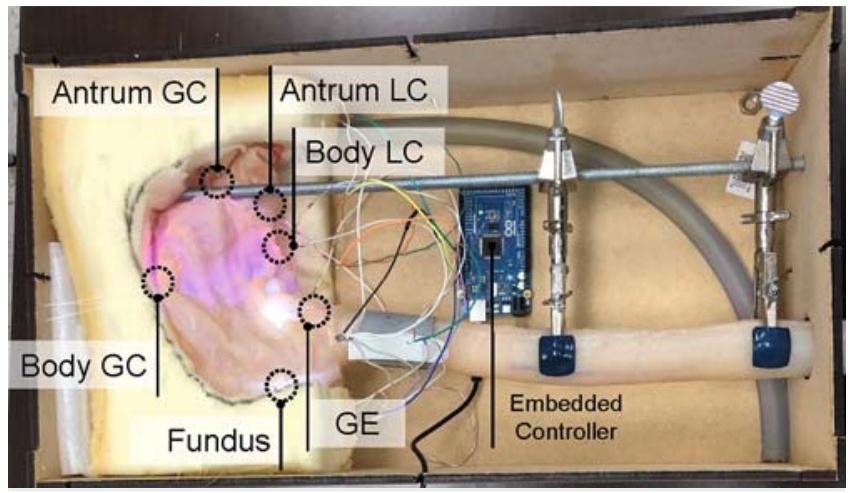

- Fig. 3 The human stomach and esophagus phantom with LED marked anatomic landmarks.

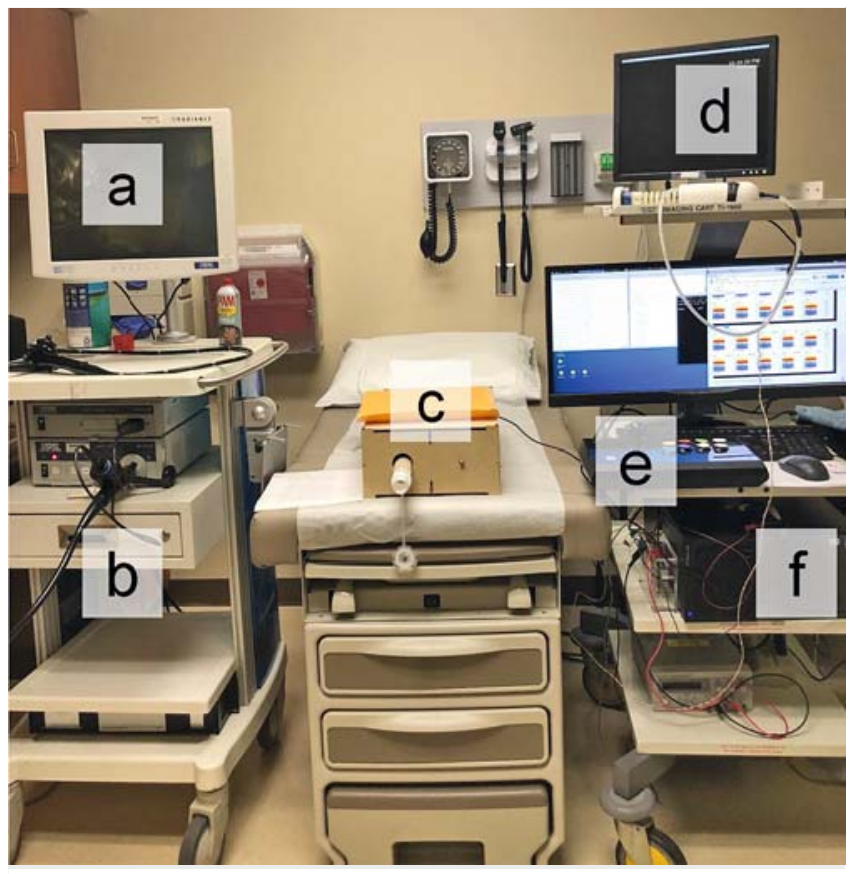

Fig. 4 Trial set-up. Conventional endoscope monitor a and tower b; stomach and esophagus phantom c; novel endoscope and monitor d; LED anatomic landmark controller e; computer for recording of trial data $\mathbf{f}$.

time $>10$ minutes was reached. $>$ Fig. 6 summarizes the protocol adopted for the study.

After all 10 exams were performed, each participant completed a validated assessment instrument for each device used (NASA Task Load Index v1.0; NASA Ames Research Center, Moffett Field, California, United States) and assigned an overall satisfaction score [10]. The NASA Task Load Index (TLX) is composed of six scales: mental demand (how mentally demanding was the task), physical demand (how physically demanding was the task), temporal demand (how hurried or rushed was the pace of the task), performance (how successful were you in accomplishing what you were asked to do), effort (how hard did you have to work to accomplish your level of performance), and frustration (how insecure, discouraged, irritated, stressed, 

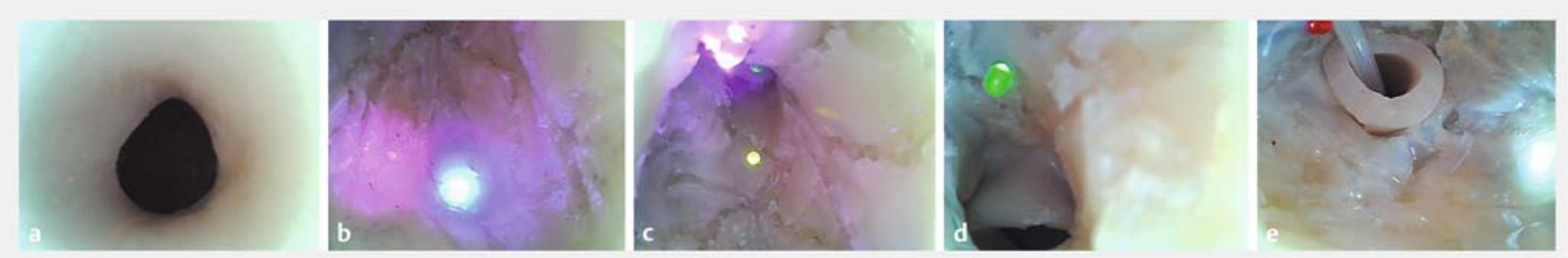

- Fig. 5 Images of the stomach and esophagus phantom taken with the novel disposable endoscope. The distal esophagus a and gastric landmarks (LED anatomic markers; b body greater curvature (blue); $\mathbf{c}$ antrum greater curvature (yellow) and body lesser curvature (purple); $\mathbf{d}$ antrum lesser curvature (green); e GEJ (red) and fundus (white) are visualized.

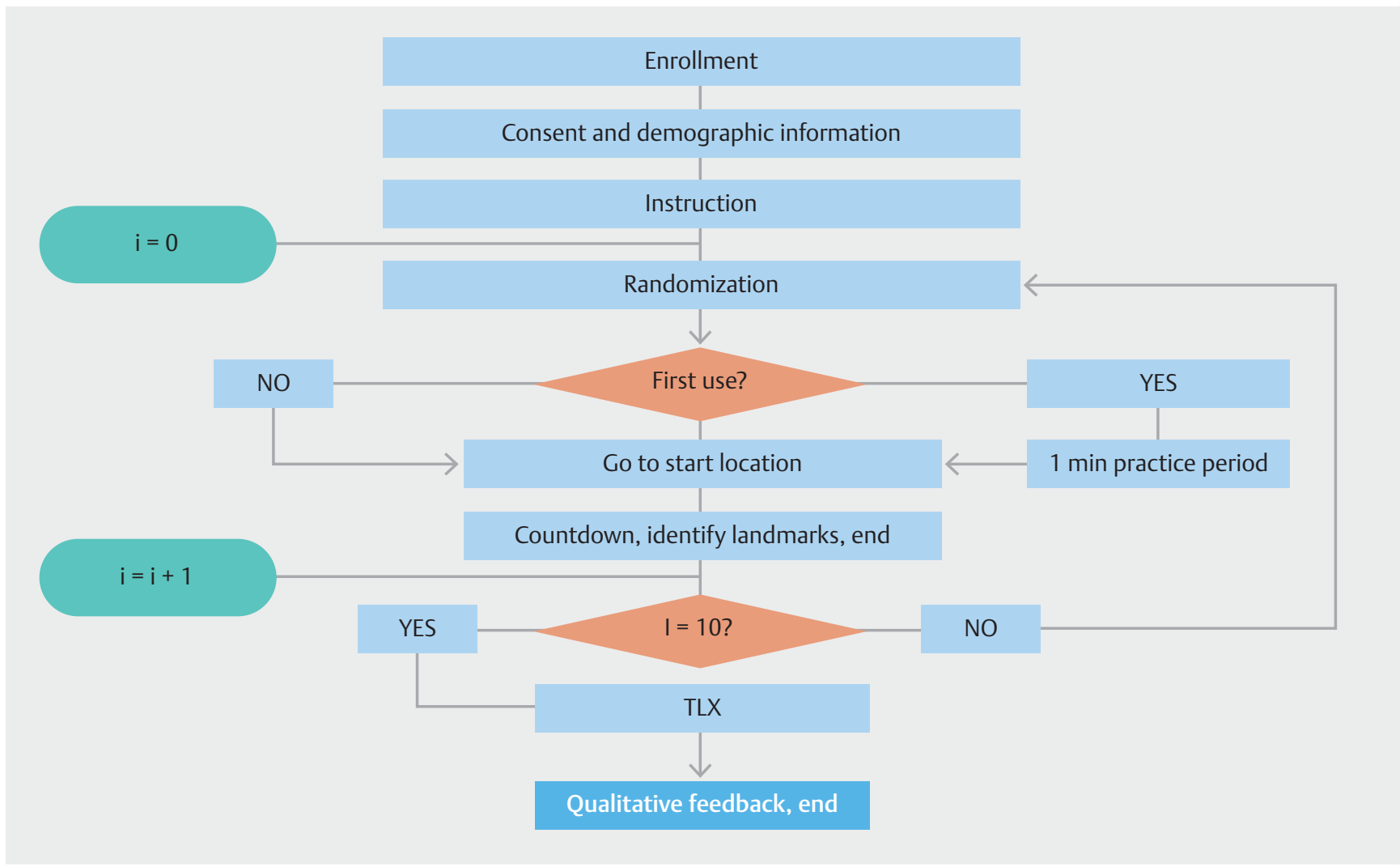

- Fig. 6 Flow diagram for the trial. Each user performed 10 endoscopies (conventional, $n=5$; novel, $n=5$ ) on the human phantom for identification of the gastric landmarks.

and annoyed were you). All subscales range from 0 (very low) to 100 (very high) with the exception of performance, which ranges from 0 (perfect) to 100 (failure). Each user was then asked for non-structured qualitative feedback using openended questions (what are your thoughts about the endoscope [conventional; novel]?).

\section{Study outcome and statistical analysis}

The main outcome measure was successful identification of the six gastric landmarks. Secondary outcomes included overall time to perform gastric landmark visualization, ease of use (TLX), and user comments. The time to visualize each individual gastric landmark and the order in which they were visualized was also recorded.
To detect differences in visualization time between devices within each user group, a paired $t$ test was used. To detect differences in visualization time for a given device between the three user groups, an ANOVA test was used. To compare the variation in total time between experienced users of conventional endoscopes when using the novel endoscope, prior data indicated a standard deviation of the difference in time of 10 seconds. With 10 experienced users (5 fellows, 5 attendings) performing five trials each with the novel endoscope, we had $90 \%$ power to detect a 5 -second difference in time. To compare the increase in time when novices used the novel endoscope, prior data indicated a standard deviation of the difference in time of 14 seconds. With 10 experienced users and five novices performing five trials each with the novel endoscope, we had 
- Table 2 Participants in the study.

\begin{tabular}{|l|l|l|l|l|}
\hline Participants & Women & Age & Lifetime \# of endoscopic procedures & Left-hand dominance \\
\hline & $\mathbf{n}(\%)$ & $\mathbf{x} \pm$ SD & $\mathbf{x} \pm$ SD & $\mathbf{n}(\%)$ \\
\hline Novice $(n=5)$ & $0(0)$ & $29 \pm 2.28$ & $0 \pm 0$ & $0(0)$ \\
\hline Fellow $(n=5)$ & $1(20)$ & $31 \pm 0.89$ & $900 \pm 300$ & $0(0)$ \\
\hline Attending $(n=5)$ & $2(40)$ & $41 \pm 2.22$ & $4800 \pm 3000$ & $1(20)$ \\
\hline All combined $(n=15)$ & $3(20)$ & $33 \pm 5.67$ & $1900 \pm 2700$ & $1(6.66)$ \\
\hline
\end{tabular}

- Table 3 Total time to complete the task of identifying all six gastric landmarks. Medians [Q1, Q3].

\begin{tabular}{|l|l|l|l|}
\hline Participant & Conventional & Novel & $P$ value \\
\hline Novice $(n=5)$ & 27.99 s $[18.07 ; 43.19]$ & 38.09 s [15.17; 64.72] & $P=0.16$ \\
\hline Fellow $(n=5)$ & 17.7 s $[11.74 ; 20.94]$ & 23.37 s $[18.45 ; 35.1]$ & $P=0.01$ \\
\hline Attending $(n=5)$ & 13.82 s $[9.62 ; 19.56]$ & 26.27 s $[17.72 ; 41.7]$ & $P<0.01$ \\
\hline All combined $(n=15)$ & 18.07 s $[11.60 ; 25.59]$ & 28.16 s $[17.62 ; 52.48]$ & $P<0.01$ \\
\hline
\end{tabular}

$90 \%$ power to detect a 6 -second difference. All calculations assumed the probability of type I error of $5 \%$.

Lower, median, and upper quartile ranges were calculated for continuous variables. A variable $Z$ was utilized to assess for differences in the order of landmark visualization for each trial. We defined $Z$ as:

$$
Z=\frac{\sum_{m} \sum_{n}\left(C_{m n}-\bar{C}\right)\left(N_{m n}-\bar{N}\right)}{\sqrt{\left(\sum_{m} \sum_{n}\left(C_{m n}-\bar{C}\right)^{2}\right)\left(\sum_{m} \sum_{n}\left(N_{m n}-\bar{N}\right)^{2}\right)}}
$$

Where $m$ and $n$ are the rows and columns of the landmarksorder matrices, respectively; $C$ identifies the landmarks-visualization order matrix when using the conventional endoscope; $N$ the landmarks-visualization order matrix using the novel endoscope; and $\bar{C}$ and $\bar{N}$ are the mean of all values of $C$ and $N$, respectively.

After completion of the trial, participants completed a validated TLX assessment instrument with overall satisfaction score for each endoscope (conventional; novel) and provided non-structured qualitative feedback by face-to-face interview. All data was securely recorded using Research Electronic Data Capture (REDCap) (Nashville, Tennessee, United States [11]. This study was approved by the medical center Institutional Review Board before initiation of data collection (IRB\#171660).

\section{Results}

Five attendings (mean number of lifetime procedures: $4800 \pm$ $3000), 5$ fellows $(900 \pm 300)$, and 5 novices $(0 \pm 0)$ completed the trial. Three of the participants were women (20\%) and one of the participants was left-hand dominant (7\%) ( $>$ Table 2). All participants $(n=15)$ successfully identified all six gastric landmarks with the conventional endoscope and with the novel endoscope for each trial $(100 \% ; n=900)$. The time to landmark identification for all groups combined was shorter with the con- ventional endoscope when compared to the novel endoscope (18.07 v. 28.16 seconds; $P<0.01$ ). The total time for all trials was normally distributed and there was no difference in time to landmark identification for the novice group when comparing the conventional endoscope to the novel endoscope (27.99 v. 38.09 seconds; $P=0.16$ ). For attendings and fellows, use of the conventional endoscope was quicker when compared to use of the novel endoscope (13.82 v. 26.27 seconds; $P<0.01$ and 17.7 v. 23.37 seconds; $P=0.01$ respectively) ( $\vee$ Table 3$)$. The maximum and minimum time to visualize all landmarks was 113.07 and 5.04 seconds with the conventional endoscope and 126.33 and 9.42 seconds with the novel endoscope. Using the conventional endoscope, attendings were quickest when compared to the other groups $(P<0.01)$. When the novel endoscope was used, there was no difference in time between user groups (attending, fellow, novice) $(P=0.094)$. - Fig. 7 shows the lower, median, and upper quartile of both total time and specific landmark identification time for each group of participants using both devices (conventional and novel endoscope).

By recording (Supplementary Table 1) the order in which each landmark was identified with a specific device (conventional/novel), we verified that the path taken to visualize the landmarks was consistent for each user group using the two devices (novel and conventional endoscope) - verifying that the order of landmark visualization did not influence the results ( $Z=0.948$ [attendings], 0.923 [fellows] 0.907 [novices]).

All participants operated the novel endoscope successfully despite having no prior experience or formal training outside of the 1-minute practice period. The novel endoscope was reliable throughout each trial without video, electric, or mechanical failure. Participants reported both low mental demand and low physical demand ( $\triangleright$ Table 4 ). Five users specifically noted intuitiveness of the novel endoscope (2 novices), ease of use ( 1 fellow), or ease to learn (1 novice, 1 attending) in their user 

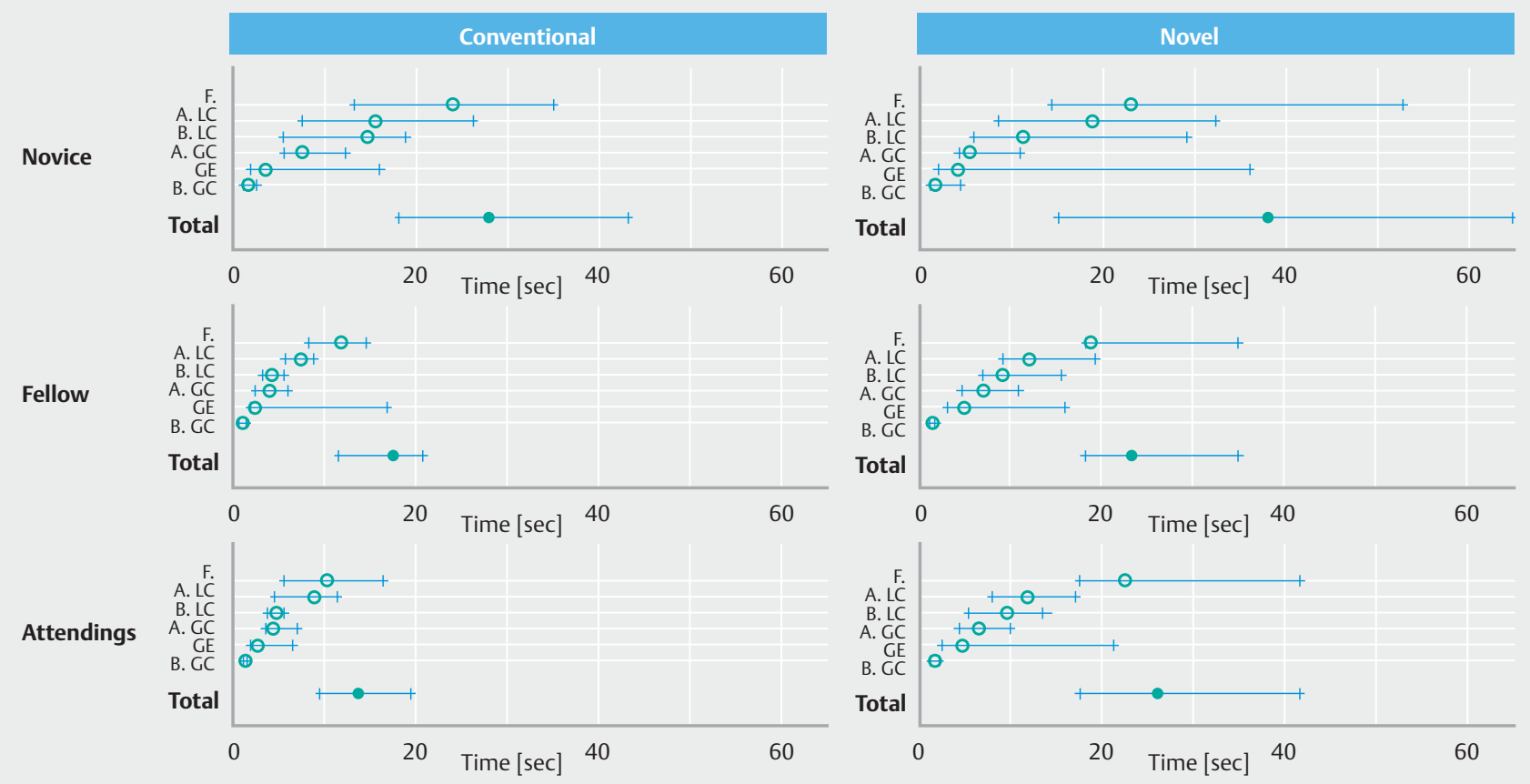

- Fig. 7 Trial results by endoscope type (conventional; novel) and user (novice; fellow; attending). Total time and landmark specific identification times are displayed (median = red circle; $Q 1 Q 3=$ blue line; $F=$ fundus; $A . L C=$ antrum lesser curvature; $B . L C=$ body lesser curvature; $A . G C=$ antrum greater curvature; B.GC= body greater curvature; $G E=$ gastroesophageal junction).

- Table 4 The NASA Task Load Index (TLX) subscale scores for the novel endoscope and conventional endoscopy by participant group. All subscales range from 0 (very low) to 100 (very high) with the exception of performance, which ranges from 0 (perfect) to 100 (failure). Median [Q1, Q3].

\begin{tabular}{|c|c|c|c|c|c|c|}
\hline \multirow[t]{2}{*}{ Subscale } & \multicolumn{2}{|c|}{ Novice $(n=5)$} & \multicolumn{2}{|c|}{ Fellow $(n=5)$} & \multicolumn{2}{|c|}{ Attending $(n=5)$} \\
\hline & Novel & Conventional & Novel & Conventional & Novel & Conventional \\
\hline Mental demand & $32[7,62]$ & $41[23,66]$ & $45[9,51]$ & $3[1,12]$ & $27[11,51]$ & $13[5,18]$ \\
\hline Physical demand & $17[5,49]$ & $42[10,82]$ & $20[14,47]$ & $10[2,29]$ & $13[10,41]$ & $12[5,20]$ \\
\hline Temporal demand & $50[35,56]$ & $63[54,76]$ & $19[12,45]$ & $4[1,35]$ & $33[13,51]$ & $23[8,35]$ \\
\hline Performance & $25[10,34]$ & $37[16,59]$ & $33[26,36]$ & $20[5,51]$ & $47[07,59]$ & $17[07,33]$ \\
\hline Effort & $50[22,73]$ & $67[33,80]$ & $25[9,55]$ & $20[6,39]$ & $34[20,58]$ & $7[5,13]$ \\
\hline Frustration & $74[24,76]$ & $51[48,71]$ & $16[13,55]$ & $9[1,32]$ & $42[12,54]$ & $8[3,14]$ \\
\hline
\end{tabular}

feedback comments (Supplementary Table2). Three users specifically expressed satisfaction with the novel platform's low weight (1 novice, 1 attending, 1 fellow) and ergonomics (1 fellow) when compared to the conventional endoscope.

\section{Discussion}

The novel esophago-gastroscope was easy to maneuver, intuitive to use, and successful at visualizing key landmarks in the stomach in a timely manner regardless of users having no prior experience with the novel endoscope. While fellows and attendings had longer mean times with the novel endoscope when compared to the conventional endoscope, their times remained within a clinically acceptable range (difference of ap- proximately 10 seconds). This finding is likely due to the fellows' and attendings' experience and comfort in using the conventional endoscope - as the mechanism of the novel endoscope is inherently different from a conventional endoscope (i. e. no need for insertion tube torque or thumb wheel manipulation of Bowden cables).

In the novice group, without any endoscopy experience, there was no such difference, suggesting that unbiased users performed with essentially equal efficiency with both devices. In addition, both experienced endoscopists and novices performed well with the novel endoscope, suggesting a short learning curve (supporting the intuitiveness of the novel platform.) This portends well for potential efficacy in clinical scenarios for diagnostic upper endoscopy given our intended de- 
mographic of non-specialized users (i.e. non-traditional endoscopists: ED providers, ICU physicians, primary care providers, etc.). Users of the novel platform may not require extensive technical training in traditional endoscopy in order to maneuver the novel endoscope, and can instead focus their attention on cognitive skill (i.e. recognition of "normal" or "abnormal" findings to support triage decisions).

All participants in the study found the novel endoscope easy to use and intuitive with low mental and physical demand. Novices had reduced temporal demand and effort with the novel endoscope. While novices reported increased frustration with the novel endoscope, this was primarily related to incidences where the pivoting pad slipped and necessitated re-establishment of pad contact with their body. While attendings and fellows favored the conventional endoscope, novices favored the novel endoscope. This is likely explained by the extensive experience with conventional endoscopy in the fellow and attending groups, who attempted to drive the novel endoscope as if it were a conventional endoscope. Novices, who lacked any experience with either endoscope, preferred the novel endoscope. Regardless of endoscopy experience, all users were able to complete the task with the novel endoscope in an efficient manner and were pleased with the novel device's ergonomics, low weight, and ease of manipulation (Supplementary Table 2). This would suggest that the intuitive drive mechanism is robust, effective, and functions well for the task at hand (especially for those without any specialized conventional endoscopy training).

Our results also suggest potential efficacy in clinical use, pending further studies and minor design revisions. This is especially significant given that the novel endoscope is lowcost (less than \$35 in materials; lower if produced on a large scale), intuitive to drive without prior experience, and without need for bulky equipment or device reprocessing. One could imagine point-of-care management decisions being potentially made in non-traditional settings (primary care provider office, inpatient medical or surgical ward) at the initial encounter. This could be possible with administration of a topical anesthetic agent given the current flexible catheter width of $7 \mathrm{~mm}$ (with plan to reduce the tip dimension to less than $8 \mathrm{~mm}$ ) with pliability similar to currently used orogastric tubes. Similar to other tools such as central line kits, providers would have the potential to take the novel device "off-the-shelf," open the package, and perform their exam to triage the patient to conventional endoscopy with intervention or another care plan. Assuming that the novel endoscope is well tolerated after application of a topical anesthetic and that the diagnostic capability sufficiently facilitates reduced utilization of conventional upper endoscopy, AEs could potentially be reduced and the risk/benefit ratio for conventional upper endoscopy could be more advantageous if it were purely an interventional procedure.

Examples of this shift in work flow that could be possible with appropriate training (technical and cognitive) for non-specialized users (i.e. to identify "normal" from "abnormal") include a primary care provider seeing a patient in clinic with long-standing GERD or GERD non-responsive to acid suppression medications, odynophagia, or suspicion of peptic ulcer or gastrointestinal etiology for anemia. In this case, a primary care provider could perform rapid bedside diagnostic examination of the patients' stomach and esophagus. Identification of any suspicious finding(s) (i.e. presumed Barrett's esophagus, esophageal stenosis, candida esophagitis, neoplasm, varices, peptic ulcer) could then be sent for an expedited and directed interventional upper endoscopy performed in the traditional setting.

Another example, with appropriate training, could be screening for esophageal and gastric varices in an outpatient hepatology clinic for "point-of-care" diagnosis. As patients with cirrhosis are at increased risk for sedation-related AEs, avoidance of conventional upper endoscopy, unless necessary to perform esophageal band ligation of varies, would be optimal [12]. In the hospital setting (ED, inpatient ward, or ICU), having immediate bedside diagnostic information, as in the case of suspected gastrointestinal bleeding or foreign body, would allow for prompt and accurate triaging of the patient (i.e. need for gastroenterology consultation versus pulmonary, interventional radiology, or ENT, admit or discharge from the $E D$, expedited inpatient travel case, transfer to an ICU).

A potential limitation of our study is the small number of participants from a single institution and single division (Gastroenterology). While we were able to detect a difference between the groups as intended based on the main outcome measure and power calculation, future studies will include an increased breadth of participants from multiple specialties (pulmonologists, emergency medicine providers, primary care providers, surgical), skill levels (PGY trainees in those specialties, mid-level providers, and attendings) and from multiple centers with a formal learning curve assessment.

Another potential limitation was the practice period with the conventional endoscope and the novel endoscope. To avoid potentially jeopardizing or influencing the assessment of ease of use, the time frame of 1 minute was selected. Our team felt that if the practice period were longer, any difference between the platforms that might exist might be undetectable. In addition, given the intended users of the novel platform (primary care providers, ICU providers, emergency department providers), we aimed to demonstrate that use of the novel endoscope was intuitive with a short learning curve that required no more than 1 minute of time to comprehend.

The path taken to visualize the landmarks had the potential to significantly affect the results - if one order or path for visualizing the landmarks was inherently quicker than another, the outcome would be more reflective of the path choice taken and less of a measure of the device itself. From careful review of the landmark visualization order for each trial for each device, there was no significant difference for all users with each device. This was also supported by our calculated Z variable.

We used a human stomach phantom with LED markings of key anatomic landmarks. This model was chosen based on its accurate anatomy, as it was created from a human patient's CT scan, and has ease of implementation in the outpatient clinic setting (i.e. no need to travel to an animal lab or other facility to perform the trials). As the model could be brought into the outpatient clinic, the set-up and arrangement of the trial was 
in alignment with the intended location for future clinical use. In addition, the model allowed for confounding factors such as food, debris, mucous, and drying out of ex-vivo tissue to be avoided - thus ensuring that all participants "saw" the same stomach under the same conditions. The LED markings also ensured that all landmarks remained in constant position for all trials for all users, eliminating this aspect of variability between trials. In addition, the LED markers were of ultra-low power and provided orders of magnitude lower illumination when compared to the illumination modules in the novel endoscope or the conventional endoscope - hence, they did not provide any meaningful enhanced illumination for assisting the camera module of the endoscope while completing the trials.

While the novel endoscope was designed to allow for users to identify normal from abnormal, after a period of technical and cognitive training, future studies will be needed to determine if users are able to definitively characterize the abnormality/pathology they have seen. thereby enhancing the novel endoscope's potential clinical utility. In addition, the tip of the novel endoscope is currently $13.5 \mathrm{~mm}$ diameter due to the use of off-the-shelf bellows (the maximum reasonable size for a patient to ingest with topical anesthetic). As a result, our team is in the process of reducing the tip diameter to less than $8 \mathrm{~mm}$ for enhanced patient comfort and ease of oral intubation now that the technology is available at the desired price point. While we initially envision oropharyngeal intubation with a topical anesthetic agent, nasopharyngeal intubation may eventually become a possibility through further reductions in tip and catheter diameter.

\section{Conclusion}

In conclusion, all users were pleased with the novel endoscope. The intuitiveness and key features of the platform have the potential to allow for rapid, low-cost, diagnostic assessment of the esophagus and stomach in non-endoscopy units or nonICU-based settings to facilitate patient management decisions, minimize equipment encumbrance, and avoid cross-contamination. This could facilitate patient care, potentially reduce risk from diagnostic procedures through possible avoidance, expedite therapeutic procedures, and improve allocation of endoscopy resources. Planning for first-in-human trials is currently underway.

\section{Acknowledgements}

Research reported in this publication was supported by the $\mathrm{Na}$ tional Institute of Biomedical Imaging and Bioengineering of the National Institutes of Health under Award Number R01EB018992 and by the National Center for Advancing Translational Sciences of the National Institutes of Health under Award Number UL1 TR000445, the Royal Society under grant number $\mathrm{CH} 160052$, the Engineering and Physical Sciences Research Council (EPSRC) under grant number EP/P027938/1, and by the Higher Education Funding Council for England (HEFCE) Global Challenge Research Fund under grant number
95543290. The content is solely the responsibility of the authors and does not necessarily represent the official views of the NIH, Royal Society, EPSRC, or the HEFCE.

The authors would like to thank William McKinney, Max Pudiak, Alexander Smith, Maggie O'Connor, Taylor Cannon, Samantha Kopinsky, and Ethan Vanderslice for the design, manufacturing, and creation of the human upper gastrointestinal tract phantom; as well as to Dr. Michael Vaezi, Director of Vanderbilt GI Clinical Enterprise, for use of the GI outpatient clinic space/examination room to complete the trials.

\section{Competing interests}

None

\section{References}

[1] Peery AF, Dellon ES, Lund J et al. Burden of gastrointestinal disease in the United States: 2012 update. Gastroenterology 2012; 5: 1179187

[2] Ben-Menachem T, Decker G, Early DS et al. Adverse events of upper GI endoscopy. Gastrointest Endosc 2012; 76: 707-718

[3] Ofstead CL, Quick MR, Eiland JE et al. A glimpse at the true cost of reprocessing endoscopes. International Association of Healthcare Central Service Materiel Management 2017: Available from https:// www.bostonscientific.com/content/dam/bostonscientific/uro-wh/ portfolio-group/LithoVue/pdfs/Sterilization-Resource-Handout.pdf Accessed 2019 March 5

[4] Faigel DO, Baron TH, Lewis B et al. Ensuring competence in endoscopy. Prepared by the ASGE/ACG Taskforce on Quality in Endoscopy 2007: Available from: https://www.asge.org/docs/default-source/ education/practice_guidelines/doc competence.pdf?sfvrsn=6 Accessed 2019 March 5

[5] Garbin N, Sarker S, Sohn DC et al. Evaluation of a novel disposable upper endoscope for unsedated bedside (non-endoscopy unit based) assessment of the upper gastrointestinal tract. Gastrointest Endosc 2017; 85: AB304

[6] Burgner-Kahrs ], Rucker DC, Choset H. Continuum robots for medical applications: a survey. IEEE Trans Robot 2015; 31: 1261-1280

[7] Simaan N. Snake-like units using flexible backbones and actuation redundancy for enhanced miniaturization. IEEE Int Conf Robot Autom 2005: $3012-3017$

[8] Bailly Y, Amirat Y. Modeling and control of a hybrid continuum active catheter for aortic aneurysm treatment. IEEE Int Conf Robot Autom 2005: $924-929$

[9] Gagarina T, Joli P. Modeling and experimental analysis of a new bellow type actuators for active catheter end-effector. ROMAN 2001: 612 617

[10] Hart SG, Staveland LE. Development of NASA-TLX (Task Load Index): Results of empirical and theoretical research. Advances Psychol 1988; 52: $139-183$

[11] Harris PA, Taylor R, Thielke R et al. Research electronic data capture (REDCap)-a metadata-driven methodology and workflow process for providing translational research informatics support. J Biomed Inform 2009; 42: $377-381$

[12] Abbas N, Makker J, Abbas $\mathrm{H}$ et al. Perioperative care of patient with liver cirrhosis: a review. Health Serv Insights 2017: 10 